\title{
Acute respiratory distress syndrome
}

\author{
Marco Confalonieri, Francesco Salton and Francesco Fabiano
}

Affiliation: Pulmonology Dept, University Hospital of Cattinara, Trieste, Italy.

Correspondence: Marco Confalonieri, S.C. Pneumologia, Ospedale di Cattinara, Strada di Fiume 447, 34149 Trieste, Italy. E-mail: marco.confalonieridasuits.sanita.fvg.it

@ERSpublications

A review of acute respiratory distress syndrome http://ow.ly/ITXq308ZLtE

Cite this article as: Confalonieri M, Salton F, Fabiano F. Acute respiratory distress syndrome. Eur Respir Rev 2017; 26: 160116 [https://doi.org/10.1183/16000617.0116-2016].

ABSTRACT Since its first description, the acute respiratory distress syndrome (ARDS) has been acknowledged to be a major clinical problem in respiratory medicine. From July 2015 to July 2016 almost 300 indexed articles were published on ARDS. This review summarises only eight of them as an arbitrary overview of clinical relevance: definition and epidemiology, risk factors, prevention and treatment. A strict application of definition criteria is crucial, but the diverse resource-setting scenarios foster geographic variability and contrasting outcome data. A large international multicentre prospective cohort study including 50 countries across five continents reported that ARDS is underdiagnosed, and there is potential for improvement in its management. Furthermore, epidemiological data from low-income countries suggest that a revision of the current definition of ARDS is needed in order to improve its recognition and global clinical outcome. In addition to the well-known risk-factors for ARDS, exposure to high ozone levels and low vitamin D plasma concentrations were found to be predisposing circumstances. Drug-based preventive strategies remain a major challenge, since two recent trials on aspirin and statins failed to reduce the incidence in atrisk patients. A new disease-modifying therapy is awaited: some recent studies promised to improve the prognosis of ARDS, but mortality and disabling complications are still high in survivors in intensive care.

\section{Definition and epidemiology}

Since its first description by Ashbaugh et al. [1] in 1967, the acute respiratory distress syndrome (ARDS) has been widely recognised as a major clinical problem worldwide, carrying a high morbidity and mortality burden [2-4]. Although the recent Berlin definition [5] is probably much better than previous ones, there is still a high variability in both epidemiology and clinical outcomes in diverse healthcare settings [4]. In fact, the incidence of ARDS ranges from 1.5 cases per 100000 [2] to nearly 79 cases per 100000 [3], with European countries reporting a lower incidence than USA [6]. Moreover, studies from Brazil reported incidence rates ranging from 1.8 to 31 per $100000[7,8]$.

Although the overall survival rate is improving [9, 10], there is a notable difference when considering in-hospital mortality over several observational studies [2-4, 8-11]. This may be explained by differences in risk factors, availability of diagnostics, ability to recognise ARDS and some selection biases affecting clinical trials [12]. Recently, a large international observational study (the LUNG SAFE trial) evaluated the incidence of ARDS across 459 intensive care units (ICUs) in 50 countries [13]. To assess the clinical recognition of ARDS according to the latest definition, any patient inclusion into the trial was made through a computer algorithm following the Berlin criteria [5], and then compared to the diagnosis made by the attending physicians. Among 4499 patients who developed acute hypoxaemic respiratory failure, ARDS occurred in $10.4 \%$ of total ICU admissions and in $23.4 \%$ of patients requiring mechanical ventilation.

Received: Dec 082016 | Accepted after revision: Jan 172017

Conflict of interest: None declared.

Provenance: Commissioned article, peer reviewed.

Copyright CERS 2017. ERR articles are open access and distributed under the terms of the Creative Commons Attribution Non-Commercial Licence 4.0. 
Higher incidence rates were reported in North America, Oceania and Europe, compared to South America, Asia and Africa. Overall, 30.0\% of patients had mild ARDS, 46.6\% moderate ARDS and the remaining 23.4\% had severe ARDS according to the Berlin criteria. The clinical recognition of ARDS was only $34 \%$ at day 1 , and $60.2 \%$ at the end of ICU stay, and was often delayed and lower in mild cases compared to severe ones [13]. Secondary end-points of the LUNG SAFE trial included ventilator management and the use of adjunctive interventions (neuromuscular blockade, prone positioning, recruiting manoeuvres, extracorporeal membrane oxygenation, inhaled vasodilators and corticosteroids). ARDS patients were disturbingly undertreated according to scientific evidence and medical practice [14-16]: the use of optimal mechanical ventilation was low ( $82.6 \%$ of patients received $<12 \mathrm{cmH}_{2} \mathrm{O}$ positive end-expiratory pressure (PEEP) and $35 \%$ were ventilated with tidal volumes $>8 \mathrm{~mL} \cdot \mathrm{kg}^{-1}$ ideal body weight without measurement of their plateau pressure) and even adjunctive treatments were underutilized (pronation $16 \%$, corticosteroids $23 \%$, recruitment manoeuvres 33\% and neuromuscular blockade 38\%) [13]. Severity of ARDS worsened in 19\% of patients, in-hospital mortality rate was $40 \%$ and mortality increased in parallel with increased driving pressure (Pplateau-PEEPextrinsic) and with ARDS severity (46\% severe ARDS) [13]. Besides some criticism about any possible source of bias in the LUNG SAFE trial [16], we fully agree with the authors' conclusion that "there is a potential for improvement in the management of ARDS patients" [13].

In a second recent article in the field of ARDS epidemiology and definition, RIviELLo et al. [17] explored the incidence of ARDS in a Rwandan university hospital holding most of the mechanical ventilators existing in a country with $>12$ million inhabitants [18]. The epidemiology of ARDS in low-income countries has never been reported before. However, this study clearly showed that the Berlin definition is not suitable in resource-constrained settings with difficult access to arterial blood gas data, chest radiography and mechanical ventilation. The authors developed a modification of the Berlin criteria to define ARDS (Kigali ARDS) [17]. Table 1 compares the Berlin [5], Kigali [17] and previous American-European consensus definition [19]. The Kigali ARDS definition maintains the same time frame of 1 week and origin of oedema criteria of the Berlin definition. The minimum PEEP requirement is removed, whereas hypoxaemia is evaluated using the ratio of arterial oxygen saturation measured by pulse oximetry $\left(\mathrm{S}_{\mathrm{PO}_{2}}\right)$ /inspiratory oxygen fraction $\left(\mathrm{FiO}_{2}\right)$ $\leqslant 315$ with $\mathrm{SpO}_{2} \leqslant 97 \%$. This criterion was based on the study by Rice et al. [20], who showed that $\mathrm{SpO}_{2} / F_{\mathrm{iO}}$ ratio values of 235 and 315 correspond, to arterial oxygen tension $/ \mathrm{FiO}_{2}$ ratios of 200 and 300, respectively, with good sensibility and specificity. Bilateral chest opacities were evaluated through the routine use of chest ultrasonography, whereas chest radiographs were considered when available. The use of ultrasonography was supported by previous evidence [21]: B-lines and/or lung consolidation without pleural effusion in at

TABLE 1 American-European Consensus Conference (AECC), Berlin and Kigali criteria for acute respiratory distress syndrome (ARDS)

\section{AECC definition Berlin criteria}

Within 1 week of a known clinical insult or new or worsening respiratory symptoms

Oxygenation

$\mathrm{PaO}_{2} / \mathrm{FiO}_{2} \leqslant 200 \mathrm{mmHg}$ ldefined as acute lung injury if $\leqslant 300 \mathrm{mmHg}$ )

PEEP requirement None

Chest imaging

Bilateral infiltrates seen on frontal chest radiograph

Origin of oedema
Pulmonary artery wedge pressure $<18 \mathrm{mmHg}$ when measured or no evidence of left atrial hypertension
Mild: $\mathrm{PaO}_{2} / \mathrm{FiO}_{2}>200 \mathrm{mmHg}$ but $\leqslant 300 \mathrm{mmHg}$ Moderate: $\mathrm{PaO}_{2} / \mathrm{FiO}_{2}>100 \mathrm{mmHg}$ but $\leqslant 200 \mathrm{mmHg}$ Severe: $\mathrm{PaO}_{2} / \mathrm{FiO}_{2} \leqslant 100 \mathrm{mmHg}$

Minimum $5 \mathrm{cmH}_{2} \mathrm{O}$ PEEP required by invasive mechanical ventilation (noninvasive acceptable for mild ARDS)

Bilateral opacities not fully explained by effusions, lobar/lung collapse or nodules by chest radiograph or CT

Respiratory failure not fully explained by cardiac failure or fluid overload (need objective assessment, such as echocardiography, to exclude hydrostatic oedema if no risk factor present)
Kigali modification of Berlin criteria

Within 1 week of a known clinical insult or new or worsening respiratory symptoms

$\mathrm{SpO}_{2} / \mathrm{FiO}_{2} \leqslant 315$

No PEEP requirement, consistent with AECC definition

Bilateral opacities not fully explained by effusions, lobar/lung collapse or nodules by chest radiograph or ultrasound

Respiratory failure not fully explained by cardiac failure or fluid overload (need objective assessment, such as echocardiography, to exclude hydrostatic oedema if no risk factor present)

PEEP: positive end-expiratory pressure; $\mathrm{PaO}_{2}$ : arterial oxygen tension; $\mathrm{FiO}_{2}$ : inspiratory oxygen fraction; $\mathrm{SpO}_{2}$ : arterial oxygen saturation measured by pulse oximetry; CT: computed tomography. 
least one field on each side of the chest were considered to be consistent with bilateral lung opacities. The Kigali study was performed prospectively over a 6-week period. Patients who were found to be hypoxaemic received lung ultrasound daily. The exclusion of cardiac failure was based on chart review and echocardiography, when available. On a global number of 1046 screened patients, 88 out of 126 hypoxic patients had a $\mathrm{SpO}_{2} / \mathrm{FiO}_{2}$ ratio of $\leqslant 315$, and $42(4 \%)$ were eventually found to have ARDS according to the Kigali definition [17]. In this African population the most common causes of ARDS were infection, surgery and trauma. Only $30.9 \%$ of patients were admitted to ICU and they were all mechanically ventilated. In the Kigali trial the median age was relatively low (37 years) and the mortality rate $50 \%$ [17]. None of these patients would have been identified if the standard Berlin definition had been used. Altogether, these data show that ARDS is still underdiagnosed and undertreated worldwide, and that the current definition of ARDS is inadequate in most clinical scenarios.

\section{Risk factors}

ARDS is not a disease, but a clinically defined condition with acute respiratory failure occurring de novo as a result of clearly determined pulmonary and nonpulmonary insults. Although most ARDS predisposing conditions are well known [3, 22, 23], there is insufficient awareness about environmental and individual risk factors. Chronic alcohol abuse and active or passive cigarette smoke have been associated with an increased incidence of ARDS [24], whereas the impact of environmental pollution on the incidence of ARDS has not been established. WARE et al. [25] studied the effect of long-term ozone exposure in patients who had a recognised risk factor for ARDS and lived within $50 \mathrm{~km}$ of one of the 163 air quality monitoring stations in a large area covering eight states around Nashville (TN, USA). Data on daily average levels of ozone, nitrogen dioxide $\left(\mathrm{NO}_{2}\right)$, sulfur dioxide and particles with a $50 \%$ cut-off aerodynamic diameter of $10 \mu \mathrm{m}$ and $2.5 \mu \mathrm{m}$ were recorded daily and long-term exposures were estimated. Long-term exposure to ozone was found to be an independent risk factor for ARDS in the entire cohort (OR 1.58, 95\% CI 1.27-1.96) and incidence of ARDS increased with increasing ozone exposure $(\mathrm{p}<0.001)$. In particular, risk of ARDS was higher in the subgroup of patients who had trauma and in current smokers. Conversely, a causative role of long-term exposure to $\mathrm{NO}_{2}$ and other pollutants was not confirmed. Notably, the recorded levels of ozone were always found to be lower than required by the US Environmental Protection Agency cut-offs. Although this study has some methodological limitations (relatively confined to single geographic region and pollutants levels not directly measured), it clearly showed that air pollution may play an important role in ARDS pathogenesis.

Another debated individual risk factor is vitamin $\mathrm{D}$, as it is involved in the modulation networks of innate and adaptive immune function [26] and its deficiency is linked to an increased risk of pneumonia [27] and sepsis [28]. A recent clinical and translational study [29] showed that vitamin D deficiency may increase the risk of ARDS. The study recruited 52 patients with ARDS (mostly caused by pneumonia and extrapulmonary sepsis), 58 patients undergoing oesophagectomy (at risk of ARDS), eight patients receiving vitamin D supplementation prior to oesophagectomy and 18 healthy controls. The plasma levels of $25(\mathrm{OH})$ $\mathrm{D}_{3}, 1,25(\mathrm{OH}) 2-\mathrm{D}_{3}$ and vitamin $\mathrm{D}$ binding protein were measured: $25(\mathrm{OH})$ vitamin $\mathrm{D}_{3}$ levels $<50 \mathrm{nmol} \cdot \mathrm{L}^{-1}$ were classified as deficient and levels $<20 \mathrm{nmol} \cdot \mathrm{L}^{-1}$ as severely deficient. Study results showed that all patients with ARDS and $96 \%$ of unsupplemented oesophagectomised patients were vitamin D deficient, compared to healthy controls. Postoperative risk of ARDS was $37.5 \%$ in severely deficient patients, whereas the risk of those with milder deficiency was as low as $15 \%$. Notably, in the oesophagectomy cohort, vitamin $\mathrm{D}$ level was the only difference between patients who developed postoperative lung injury and those who did not. Plasma levels of $1,25(\mathrm{OH}) 2 \mathrm{D}_{3}$ were lower in ARDS patients admitted to ICU who died and in oesophagectomised patients who developed ARDS. Severe vitamin D deficiency was associated with increased markers of alveolar damage, such as extravascular lung water index and pulmonary vascular permeability index, on post-operative day 1 , but these alterations were mitigated in patients who were supplemented with vitamin $\mathrm{D}_{3}$ prior to oesophagectomy. This study suggests that vitamin $\mathrm{D}$ deficiency is ubiquitous among ARDS patients, and is related to adverse outcome as it takes part in the pathophysiological mechanisms involved in alveolar permeability and inflammatory responses. In conclusion, there is a potential preventive and therapeutic role of vitamin D supplementation in ARDS. Nevertheless, both high ozone exposure and vitamin D deficiency need confirmation as risk factors, and the mechanisms behind their contribution to the development of ARDS have to be better understood.

\section{Prevention}

The median time to the onset of ARDS is 2 days after hospital admission [30]. As such, the opportunity for ARDS prevention is constrained in a small window of time [31]. Some observational studies and experimental data suggested a potential preventive role of antiplatelet therapy in patients at high risk of ARDS [32-34]. As ARDS pathogenesis includes a series of dysregulated mechanisms (e.g. maladaptive 
platelet activation) it may be deduced that drugs targeted towards these mechanisms would be effective in ARDS prevention and treatment.

The preventive role of aspirin and statins has been re-evaluated in two studies [35, 36]. Kor et al. [35] studied the role of early administration of aspirin in patients at risk of ARDS in a multicentre, double-blind, placebo-controlled, randomised study. 400 adult patients at risk of ARDS according to a lung injury prediction score [37] $\geqslant 4$ were randomised to receive either aspirin at a loading dose of $325 \mathrm{mg}$ within $24 \mathrm{~h}$ of admission to the emergency department followed by $81 \mathrm{mg}$ daily for 7 days, or placebo. Most patients had suspected sepsis or pneumonia as a risk factor. Primary outcome was development of ARDS according to the Berlin criteria. Overall, 37 (9.5\%) patients developed ARDS, of whom 20 (10.3\%) were in the aspirin group and $17(8.7 \%)$ were in the placebo group. Secondary outcomes, including ventilator-free days to hospital day 28 , ICU and hospital lengths of stay, 28-day and 1-year mortality and incidence of adverse events did not differ between the two groups [35].

As for statins, a recent meta-analysis [36] evaluated 13 studies (four randomised clinical trials (RCTs) and nine cohort studies), published between 2007 and 2015 and covering a total of 12145 patients at high risk of ARDS or diagnosed with acute lung injury/ARDS. Simvastatin and rosuvastatin were most frequently used. Quality assessment graded RCTs and cohort studies as high and moderate, respectively. $>9835$ patients at high risk of ARDS were enrolled in a single RCT and five cohort studies, showing in the former that the incidence of ARDS did not differ between statins and placebo groups $(p=0.10)$, and in the latter that previous use of statins did not decrease the morbidity of ARDS. Data from 3159 patients included in three RCTs and six cohort studies showed that statins did not influence ARDS mortality. Similar results were reported for ventilator-free days, oxygenation index, ICU and length of hospital stay. The negative results of this meta-analysis are undeniable, although some limitations must be taken into account: the high heterogeneity in the analysis of morbidity and length of stay in the cohort studies, the different molecules and duration of statin therapy and the short follow-up time. Nevertheless, both aspirin and statins failed to prevent ARDS in randomised trials with different timings of intervention. The timing of intervention remains to be a major challenge of ARDS prevention trials. However, to date, it seems that ARDS is not preventable.

\section{Treatment}

Treatment of ARDS is mainly supportive, and it encompasses all measures depicted in table 2. In particular, invasive mechanical ventilation with lung protective strategies is the mainstay of ARDS treatment, although long-term intubation may carry a high rate of complications, such as ventilator-associated pneumonia (VAP), delirium and critical illness myopathy and neuropathy. Noninvasive ventilation (NIV) and the

TABLE 2 Management of acute respiratory distress syndrome (ARDS)

\section{Management of hypoxaemia}

Supplemental oxygen

Inflammation management (corticosteroids)

Fluid management

Prone positioning [38]

Decrease oxygen consumption

Increase oxygen delivery
Intubation/mechanical ventilation (most patients)

Noninvasive ventilation for mild ARDS or to $\downarrow$ intubation rates (helmet better than face mask) [34]

Prolonged low doses $\left(1 \mathrm{mg} \cdot \mathrm{kg}^{-1} \cdot \mathrm{day}^{-1}\right)$ methylprednisolone treatment accelerates ARDS resolution and improves several clinical outcomes [39]

Aim for central venous pressure $<4 \mathrm{mmHg}$ or PAOP $<8 \mathrm{mmHg}$ to $\downarrow$ pulmonary oedema $[40,41]$

Antipyretics, sedatives, analgesics and paralysis agents [42]

Inotropics to $\uparrow$ filling pressure (if no pulmonary oedema)

Restrict transfusions to maintain haemoglobin to $7-9 \mathrm{~g} \cdot \mathrm{dL}^{-1}[43,44]$

Inhaled vasodilators (nitric oxide, prostacyclin and prostaglandin E1)

to $\uparrow V^{\prime} / Q^{\prime}$ matching [45]

Sedation and analgesia [46]

Neuromuscular blockade if severe ARDS [47]

Haemodynamic monitoring/management via CVC

Nutritional support (enteral)

Glucose control

VAP prevention and treatment [48]

DVT prophylaxis

Gastrointestinal (stress ulcers) prophylaxis

PAOP: pulmonary arterial occlusion pressure; $V^{\prime} / Q^{\prime}$ : ventilation/perfusion; CVC: central venous catheter; VAP: ventilator-associated pneumonia; DVT: deep vein thrombosis. 
application of continuous positive airway pressure (CPAP) is contemplated in mild ARDS, although its use in acute hypoxaemic respiratory failure remains controversial and the choice of the interface device is still debated [39, 40, 49].

To address some of these issues, PATEL et al. [41] evaluated the use of helmet versus face mask in a randomised single-centre trial in adult patients requiring face mask NIV for $\geqslant 8 \mathrm{~h}$. Patients were randomised either to continue face mask NIV with a single limb circuit ventilator (control group) or switched to helmet ventilation (intervention group) through an ICU ventilator in pressure support or CPAP mode. Of 740 screened patients, 83 were randomised to the face mask group or to the helmet group. Most patients were immunocompromised by virtue of cancer or transplantation and one-third had pneumonia, in both groups.

Compared to the control group, helmet-ventilated patients received higher PEEP values and lower $\mathrm{FiO}_{2}$, showing a more pronounced reduction of respiratory rate. The primary outcome (reduced intubation rate) was reached more significantly in the intervention group $(61.5 \%$ versus $18.2 \%$, absolute difference $-43.3 \%$; $\mathrm{p}<0.001)$. Persistence of respiratory failure despite the noninvasive ventilatory support was the most frequent cause of intubation in the control group, whereas deterioration of neurological status determined most of the intubations in the intervention group. Secondary outcomes (i.e. ventilator-free days, length of ICU stay, 90-day mortality, but not hospital length of stay), were lower in the helmet group (for 90-day mortality $56.4 \%$ versus $34.1 \%$, absolute difference $-22.3 \%$; $\mathrm{p}=0.02$ ). Interest in this study [41] arises from the demonstration that an effective NIV may be delivered by helmet even in moderate-to-severe ARDS. This notwithstanding, some limitations should be taken into account because of the different ventilation devices used in the two groups, the unblinded nature of the study and the early conclusions based on predetermined efficacy criteria [38]. All these possible biases, together with the difficulty to extend a single-centre experience may lead to overestimation of the outcome differences between groups.

Another adjunctive treatment for ARDS patients under permanent debate in the past two decades is the use of systemic glucocorticoids. Dysregulated pulmonary and systemic inflammation play an important role in the pathogenesis and progression of ARDS [42, 43]. It has been established in experimental and clinical studies that prolonged corticosteroid use may positively modify altered inflammatory pathways and favour pulmonary repair. However, the results of randomised trials and meta-analyses are in conflict, mainly because of the different therapeutic schemes used in different studies (different drugs, dosing, duration and timing of treatment initiation and tapering) [44, 46, 47]. However, consensus exists that if glucocorticoid treatment is to be initiated, it should be initiated before day 14 of ARDS [44].

MEduri et al. [48] performed a meta-analysis consisting of two sets of analyses. The first was an individual patient data (IPD) analysis [45] of four RCTs which investigated prolonged ( $\geqslant 7$ days) methylprednisolone treatment $1-2 \mathrm{mg} \cdot \mathrm{kg}^{-1} \cdot \mathrm{day}^{-1}$ in 322 patients (early initiation within $72 \mathrm{~h}$ in 118 patients, and later after 5-7 days in 204 patients) with an intention-to-treat methodology. The second set of analyses consisted of a trial-level meta-analysis of data from IPD analysis and additional data from four RCTs that had investigated the use of a 7-day course of hydrocortisone in early ARDS, overall including 569 patients. In IPD analysis the primary outcome was successful removal of mechanical ventilation by study day 28 . Compared to the placebo group, the methylprednisolone group had fewer patients who died before achieving unassisted breathing (12\% versus $29 \%)$, more patients who achieved unassisted breathing $(80 \%$ versus $50 \%$ ), fewer patients alive on day 28 who had remained on initial mechanical ventilation ( $8 \%$ versus $21 \%)$ and more patients discharged alive from the ICU (75\% versus $49 \%$ ) (all p-values <0.001). Patients in the methylprednisolone group achieved unassisted breathing earlier and had a shorter duration of mechanical ventilation. No clear differences between early versus late treatment initiation was noted, whereas patients undergoing a rapid tapering of the study drug after the patient achieved unassisted breathing showed a greater risk of returning to mechanical ventilation. Secondary outcomes included mechanical ventilation-free days, ICU-free days, hospital mortality, time to death by hospital discharge or by day 28 and infectious complications [48]. These data favoured steroid treatment, with a high degree of evidence for the duration of initial assisted breathing and ventilator-free day outcomes, in particular. In the trial-level meta-analysis, glucocorticoid treatment was associated with an increase in mechanical ventilation-free and ICU-free days and with a reduced risk of in-hospital mortality (total mortality risk ratio 0.52 , 95\% CI 0.33-0.82) [48]. Risk of nosocomial infection did not increase with glucocorticoid treatment. Notwithstanding the lack of large randomised clinical trials, this meta-analysis reinforces the role of prolonged low dose and slowly tapered glucocorticoids in the management of ARDS.

\section{Conclusions}

In the past year, several interesting papers were published on epidemiology, novel risk factors, prevention and treatment of ARDS. Despite the well-established advances in its supportive treatment, ARDS remains an oftentimes misdiagnosed syndrome, carrying a high burden in terms of patient morbidity and mortality, as well as healthcare costs. Even if plentiful literature exists on the pathophysiology and treatment of this 
syndrome in human and animal models, implications in clinical practice are still poor. Future directions of research should focus on identification of the mechanisms of susceptibility, primary prevention and early treatment, as well as on targeted pharmacological therapies for this devastating condition.

\section{References}

1 Ashbaugh DG, Bigelow DB, Petty TL, et al. Acute respiratory distress in adults. Lancet 1967; 2: 319-323.

2 Brun-Buisson C, Minelli C, Bertolini G, et al. Epidemiology and outcome of acute lung injury in European intensive care units. Results from the ALIVE study. Intensive Care Med 2004; 30: 51-61.

3 Rubenfeld GD, Caldwell E, Peabody E, et al. Incidence and outcomes of acute lung injury. N Engl J Med 2005; 353: 1685-1693.

4 Villar J, Blanco J, Añón JM, et al. The ALIEN study: incidence and outcome of acute respiratory distress syndrome in the era of lung protective ventilation. Intensive Care Med 2011; 37: 1932-1941.

5 Ranieri VM, Rubenfeld GD, Thompson BT, et al. Acute respiratory distress syndrome: the Berlin Definition. JAMA 2012; 307: 2526-2533.

6 Villar J, Blanco J, Kacmarek RM. Current incidence and outcome of the acute respiratory distress syndrome. Curr Opin Crit Care 2016; 22: 1-6.

7 Azevedo LC, Park M, Salluh JI, et al. Clinical outcomes of patients requiring ventilatory support in Brazilian intensive care units: a multicenter, prospective, cohort study. Crit Care 2013; 17: R63.

8 Caser EB, Zandonade E, Pereira E, et al. Impact of distinct definitions of acute lung injury on its incidence and outcomes in Brazilian ICUs: prospective evaluation of 7,133 patients. Crit Care Med 2014; 42: 574-582.

9 Cochi SE, Kempker JA, Annangi S, et al. Mortality trends of acute respiratory distress syndrome in the United States from 1999-2013. Ann Am Thorac Soc 2016; 13: 1742-1751.

10 Erickson SE, Martin GS, Davis JL, et al. Recent trends in acute lung injury mortality: 1996-2005. Crit Care Med 2009; 37: 1574-1579.

11 Sigurdsson MI, Sigvaldason K, Gunnarsson TS, et al. Acute respiratory distress syndrome: nationwide changes in incidence, treatment and mortality over 23 years. Acta Anaesthesiol Scand 2013; 57: 37-45.

12 Sjoding MW, Cooke CR, Iwashyna TJ, et al. Acute respiratory distress syndrome measurement error. Potential effect on clinical study results. Ann Am Thorac Soc 2016; 13: 1123-1128.

13 Bellani G, Laffey JG, Pham T, et al. Epidemiology, patterns of care, and mortality for patients with acute respiratory distress syndrome in intensive care units in 50 countries. JAMA 2016; 315: 788-800.

14 Wright BJ. Lung-protective ventilation strategies and adjunctive treatments for the emergency medicine patient with acute respiratory failure. Emerg Med Clin North Am 2014; 32: 871-887.

15 Baron MR, Levy BD. Recent advances in understanding and treating ARDS. F1000Res 2016; 5: 725.

16 Villar J, Schultz MJ, Kacmarek RM. The LUNG SAFE: a biased presentation of the prevalence of ARDS! Crit Care 2016; 20: 108 .

17 Riviello ED, Kiviri W, Twagirumugabe T, et al. Hospital incidence and outcomes of the acute respiratory distress syndrome using the Kigali modification of the Berlin Definition. Am J Respir Crit Care Med 2016; 193: 52-59.

18 Kwizera A, Dünser MW. A global perspective on acute respiratory distress syndrome and the truth about hypoxia in resource-limited settings. Am J Respir Crit Care Med 2016; 193: 5-7.

19 Bernard GR, Artigas A, Brigham KL, et al. The American-European Consensus Conference on ARDS. Definitions, Mechanisms, relevant outcomes, and clinical trial coordination. Am J Respir Crit Care Med 1994; 149: 818-824.

20 Rice TW, Wheeler AP, Bernard GR, et al. Comparison of the $\mathrm{SpO}_{2} / F_{\mathrm{IO}_{2}}$ ratio and the $\mathrm{PaO}_{2} / F \mathrm{FO}_{2}$ ratio in patients with acute lung injury or ARDS. Chest 2007; 132: 410-417.

21 Lichtenstein D, Goldstein I, Mourgeon E, et al. Comparative diagnostic performances of auscultation, chest radiography, and lung ultrasonography in acute respiratory distress syndrome. Anesthesiology 2004; 100 : 9-15.

22 Matthay MA, Ware LB, Zimmerman GA. The acute respiratory distress syndrome. J Clin Invest 2012; 122: 2731-2740.

23 Moss M, Bucher B, Moore FA, et al. The role of chronic alcohol abuse in the development of acute respiratory distress syndrome in adults. JAMA 1996; 275: 50-54.

24 Calfee CS, Matthay MA, Eisner MD, et al. Active and passive cigarette smoking and acute lung injury after severe blunt trauma. Am J Respir Crit Care Med 2011; 183: 1660-1665.

25 Ware LB, Zhao Z, Koyama T, et al. Long-term ozone exposure increases the risk of developing the acute respiratory distress syndrome. Am J Respir Crit Care Med 2016; 193: 1143-1150.

26 Hewison M. An update on vitamin D and human immunity. Clin Endocrinol 2012; 76: 315-325.

27 Quraishi SA, Bittner EA, Christopher KB, et al. Vitamin D status and community-acquired pneumonia: results from the third National Health and Nutrition Examination Survey. PLoS One 2013; 8: e81120.

28 Upala S, Sanguankeo A, Permpalung N. Significant association between vitamin D deficiency and sepsis: a systematic review and meta-analysis. BMC Anesthesiol 2015; 15: 84.

29 Dancer RCA, Parekh D, Lax S, et al. Vitamin D deficiency contributes directly to the acute respiratory distress syndrome (ARDS). Thorax 2015; 70: 617-624.

30 Gajic O, Dabbagh O, Park PK, et al. Early identification of patients at risk of acute lung injury: evaluation of lung injury prediction score in a multicenter cohort study. Am J Respir Crit Care Med 2011; 183: 462-470.

31 Ruthman CA, Festic E. Emerging therapies for the prevention of acute respiratory distress syndrome. Ther Adv Respir Dis 2015; 9: 173-187.

32 Chen W, Janz DR, Bastarache JA, et al. Prehospital aspirin use is associated with reduced risk of acute respiratory distress syndrome in critically ill patients: a propensity-adjusted analysis. Crit Care Med 2015; 43: 801-807.

33 Erlich JM, Talmor DS, Cartin-Ceba R, et al. Prehospitalization antiplatelet therapy is associated with a reduced incidence of acute lung injury: a population-based cohort study. Chest 2011; 139: 289-295.

34 Jin SW, Zhang L, Lian QQ, et al. Posttreatment with aspirin-triggered lipoxin $\mathrm{A}_{4}$ analog attenuates lipopolysaccharide-induced acute lung injury in mice: the role of heme oxygenase-1. Anesth Analg 2007; 104: 369-377.

35 Kor DJ, Carter RE, Park PK, et al. Effect of aspirin on development of ARDS in at-risk patients presenting to the emergency department: the LIPS-A randomized clinical trial. JAMA 2016; 315: 2406-2414. 
36 Xiong B, Wang C, Tang J, et al. Statins for the prevention and treatment of acute lung injury and acute respiratory distress syndrome: a systematic review and meta-analysis. Respirology 2016; 21: 1026-1033.

37 Gajic O, Dabbagh O, Park PK, et al. Early identification of patients at risk of acute lung injury: evaluation of lung injury prediction score in a multicenter cohort study. Am J Respir Crit Care Med 2011; 183: 462-470.

38 Beitler JR, Owens RL, Mahotra A. Unmasking a role for noninvasive ventilation in early acute respiratory distress syndrome. JAMA 2016; 315: 2401-2403.

39 Antonelli M, Conti G, Moro ML, et al. Predictors of failure of noninvasive positive pressure ventilation in patients with acute hypoxemic respiratory failure: a multi-center study. Intensive Care Med 2001; 27: 1718-1728.

40 Antonelli M, Conti G, Esquinas A, et al. A multiple-center survey on the use in clinical practice of noninvasive ventilation as a first-line intervention for acute respiratory distress syndrome. Crit Care Med 2007; 35: 18-25.

41 Patel BK, Wolfe KS, Pohlman AS, et al. Effect of noninvasive ventilation delivered by helmet $v s$ face mask on the rate of endotracheal intubation in patients with acute respiratory distress syndrome: a randomized clinical trial. JAMA 2016; 315: 2435-2441.

42 Meduri GU, Kohler G, Headley S, et al. Inflammatory cytokines in the BAL of patients with ARDS: persistent elevation over time predicts poor outcome. Chest 1995; 108: 1303-1314.

43 Baughman RP, Gunther KL, Rashkin MC, et al. Changes in the inflammatory response of the lung during acute respiratory distress syndrome: prognostic indicators. Am J Respir Crit Care Med 1996; 154: 76-81.

44 Marik PE, Pastores S, Annane D, et al. Recommendations for the diagnosis and management of corticosteroid insufficiency in critically ill adult patients: consensus statements from an international task force by the American College of Critical Care Medicine. Crit Care Med 2008; 36: 1937-1949.

45 Riley RD, Lambert PC, Abo-Zaid G. Meta-analysis of individual participant data: rationale, conduct, and reporting. $B M J$ 2010; 340: c221.

46 Confalonieri M, Urbino R, Potena A, et al. Hydrocortisone infusion for severe community-acquired pneumonia: a preliminary randomized study. Am J Respir Crit Care Med 2005; 171: 242-248.

47 Ruan SY, Lin HH, Huang CT, et al. Exploring the heterogeneity of effects of corticosteroids on acute respiratory distress syndrome: a systematic review and meta-analysis. Crit Care 2014; 18: R63.

48 Meduri GU, Bridges L, Shih M-C, et al. Prolonged glucocorticoid treatment is associated with improved ARDS outcomes: analysis of individual patients' data from four randomized trials and trial-level meta-analysis of the updated literature. Intensive Care Med 2016; 42: 829-840.

49 Thille AW, Contou D, Fragnoli C, et al. Non-invasive ventilation for acute hypoxemic respiratory failure: intubation rate and risk factors. Crit Care 2013; 17: R269. 